\title{
Practice Bulletin of the American College of Obstetrics and Gynaecologists 2019 on Magement of Hypertensive Disorders in Pregnancy- A Short Review of the Current Recommendations
}

\author{
N Popovski ${ }^{1}$ and A Nikolov ${ }^{2 *}$ \\ ${ }^{1}$ Department of Obstetrics and Gynecology, Medical University, Pleven, Bulgaria \\ ${ }^{2}$ Institute for Scientific Research, Medical University, Pleven, Bulgaria \\ *Corresponding author: A Nikolov, Institute for Scientific Research, Medical University, Pleven, Bulgaria
}

\begin{abstract}
ARTICLE INFO
Received: 幽 November 15, 2019

Published: 幽 November 22, 2019

Citation: N Popovski, A Nikolov. Practice Bulletin of the American College of Obstetrics and Gynaecologists 2019 on Magement of Hypertensive Disorders in Pregnancy- A Short Review of the Current Recommendations. Biomed J Sci \& Tech Res 23(2)-2019. BJSTR. MS.ID.003861.

\section{ABSTRACT}

The Working Group on "Pregnancy and Cardiovascular Diseases" part of The American College of Obstetricians and Gynecologists (ACOG) recently published a new practice bulletin on 23 April 2019 (ACOG No212 Practice Bulletin) in Obstetrics \& Gynecology 2019, which is the official journal of ACOG. The updated guideline provides an information about gestational hypertension treatment with an emphasis on the management of acute hypertensive disorders as well as recommendations for the treatment of pain postpartum in patients with arterial hypertension. The practical bulletin also discusses the different strategies for delivery, the use of antihypertensive drugs and upper limit normal values of blood pressure (BP). A special chapter gives a light of the treatment of preeclampsia with HELLP syndrome (hemolysis, elevated liver enzymes, low platelet count), optimal treatment of eclampsia. Treatment of hypertension and headache postpartum has been discussed too.
\end{abstract}

Keywords:Pregnancy and Heart Disease; Practice Bulletin of American College of Obstetricians and Gynecologists 2019

\section{Introduction}

In the current review we would like to give our comments on the most important aspects of NEWSLETTER No.212/2019 clinical behaviour "Pregnancy and Cardiovascular Disease". The Working Group for the Study of pregnancy and hypertension was established in 2011 as part of the presidential initiative of ACOG, which focuses on pre-eclampsia and hypertension during pregnancy. The Working Group is chaired by Dr. James M. Roberts of the University of Pittsburgh and includes 17 internationally recognized experts in hypertension during pregnancy. Bulletin No.212/2019 clarifies the classification system for hypertensive disorders in pregnancy, identifying problems in the treatment of pre-eclampsia, eclampsia and chronic hypertension in pregnancy that deserve special attention and emphasizes the potential impacts of the conditions later in life of the patient.
Preeclampsia, eclampsia and HELLP syndrome affect many women and lead to a high percentage of maternal and perinatal complications [1]. It is important for clinicians to accurately diagnose the conditions to prevent any adverse outcome. In most cases, classic presentation with hypertension and proteinuria in pregnancy>20 g.w. is observed. However, recent studies show that some women develop preeclampsia without showing any classic symptoms. Atypical cases are those that may develop before 20g.w. or with signs and symptoms of preeclampsia without hypertension or proteinuria, or those with mild abnormalities in laboratory tests [2].

In the countries of South America and those in the Caribbean hypertensive disorders account for almost $26 \%$ of deaths among mothers, while in Africa and Asia contribute about $9 \%$ of deaths [3]. 
Although maternal mortality is significantly lower in high-income countries than in developing countries, $16 \%$ of maternal mortality may be due to hypertensive disorders. In the United States, the rate of preeclampsia has increased by 25\% between 1987 and 2004 [4]. Moreover, compared to women who gave birth in 1980, those who gave birth in 2003 were 6.7 times more likely to have complications from severe preeclampsia. Treatment of preeclampsia requires many resources: for example, in 2016 in the United States treatment cost of preeclampsia during the first 12 months after delivery are worth 2.18 billion dollars (1.03 billion dollars for women and 1.15 billion dollars for new borns together).

\section{Data from Practice Bulletin of the American College of} Obstetrics and Gynaecologists 2019

\section{Recommendations for Therapeutic Strategy in Gesta- tional Hypertension/ Preeclampsia}

a) In women with gestational hypertension or preeclampsia at 37 weeks gestation or later without severe pathology delivery is recommended.

b) In patients with severe gestational hypertension or preeclampsia/eclampsia Magnesium sulfate should be started for prevention of seizures.

c) Patients should receive low dose aspirin (81mg/day) for prevention of preeclampsia between 12 and 28 g.w., if a highrisk factors for pre-eclampsia exist, such as:

I. Multiple pregnancy

II. Previous pregnancy with preeclampsia

III. kidney disease

IV. Autoimmune disease

V. Type 1 or type 2 diabetes mellitus

VI. Chronic hypertension

VII. Or more than one moderate risk factor, such as family history of preeclampsia, maternal age over 35, first pregnancy, BMI>30, specific socio-demographic characteristics.

d) NSAIDs are thought to be advantageous over opioid analgesics.

\section{Recommendations for Therapeutic Strategy in Chronic Hypertension}

a) Pregnant women with chronic hypertension should also receive low-dose aspirin between 12 and 28 g.w.

b) Antihypertensive treatment should be started in women with chronic persistent hypertension with Systolic blood pressure $\geq 150 \mathrm{mmHg}$ and/or diastolic blood pressure $\geq 95$ mmHg or at gestational hypertension, chronic hypertension with superposed gestational hypertension or hypertension with subclinical organ damage with systolic blood pressure $\geq$ $140 \mathrm{mmHg}$ and /or diastolic pressure $\geq 90 \mathrm{mmHg}$.

c) It is possible to start antihypertensive treatment even in patients with lower values of blood pressure according to the ongoing diseases or if there is an evidence of renal dysfunction.

d) ACOG recommends that pregnant women with arterial hypertension to be treated according to recently revised criteria of the American College of Cardiology (ACC) and American Heart Association (AHA), which classifies arterial hypertension in the following categories:

I. Normal Systolic BP $\leq 120 \mathrm{mmHg}$; Diastolic BP $\leq 80$ mmHg.

II. Increased Systolic BP 120-129 mm Hg; Diastolic BP $\geq 80$ $\mathrm{mmHg}$.

III. Stage 1 hypertension - Systolic BP 130-139 mmHg; Diastolic BP 80-89 mmHg.

IV. Stage 2 hypertension - Systolic BP $\geq 140 \mathrm{mmHg}$; Diastolic $\mathrm{BP} \geq 90 \mathrm{mmHg}$.

Those guidelines show that the range for normal blood pressure of not pregnant women has a lower threshold for diagnosis of hypertension in comparison with the criteria of ACOG. This is likely to lead to an overall increase in patients classified with chronic hypertension and will require co-decision by the obstetrician and cardiologist, as well as by the patient regarding appropriate guideline during pregnancy [5].

\section{Summary of the most important recommendations of the Working Group on Hypertension in Pregnancy:}
a) Careful and complete patient's history taking to assess risk factors.

b) Vitamin C or Vitamin E are not recommended to prevent preeclampsia.

c) A daily low dose of aspirin for prevention of preeclampsia is recommended for very high risk patients.

d) Initiation of an antihypertensive medicament is recommended for severe hypertension during pregnancy.

e) The decision for delivery should not be based on severity of proteinuria

f) The use of magnesium sulfate is recommended for severe preeclampsia, eclampsia or HELLP syndrome.

According to the report of the working group on ACOG of hypertension in pregnancy the diagnosis preeclampsia still requires quantitative measurement of protein in urine. The work group recommends that clinicians should also consider other factors, in addition to high blood pressure to diagnose preeclampsia without delay [6]. 
The incidence of preeclampsia in the USA has markedly increased with $25 \%$ over the past two decades and it is the leading cause of maternal and neonatal morbidity and mortality. Preeclampsia is a serious condition that usually begins after 20 g.w., and high arterial pressure is a major factor. Women who have chronic hypertension and have had preeclampsia during a previous pregnancy of 35 years or more, who have more than one fetus, have diabetes or renal disease, are with obesity, Afro-American have an autoimmune disorders are at increased risk of developing of pre-eclampsia. Signs of preeclampsia include severe headache, vision disorders, rapid weight gain, abdominal pain and swelling. Definitive treatment of preeclampsia is delivery [7].

Preeclampsia affects many women (about 5\% of pregnant women) and there are so many potentially dangerous complications, including preterm birth, severe hypertension, stroke and seizureseven mortality. Early detection and adequate treatment are a socially significant problem. According to the Working Group on the Study of Preeclampsia, it leads to a number of future cardiovascular and metabolic diseases. Women with a history of pre-eclampsia have a 1.5 to 2 times higher risk of developing heart disease later in life. Although, preeclampsia causes no direct cardiac disease, both states have common risk factors.

Women who have had hypertension or pre-eclampsia in a previous pregnancy have a higher than normal recurrence rate for both conditions in a subsequent pregnancy. Depending on which stage in the pregnancy occurred and whether the woman is overweight or have diabetes, a renal disease or other forms of vascular disease, the risk of recurrence can reach up to $50 \%$. Unfortunately, The Working Group for the Study of preeclampsia is having not still detected any effective screening test for prognosis of preeclampsia development.

\section{Highlights of 2019 Task of Working Group for Hypertension During Pregnancy:}

a) Careful and complete history taking to assess risk factors.

b) Vitamin C or Vitamin E are not recommended to prevent preeclampsia

c) Daily low-dose aspirin for prevention of pre-eclampsia is recommended for women who are with higher risk with a history of pre-eclampsia and premature birth.

d) Antihypertensive medicament is recommended for systolic blood pressure $\geq 150 \mathrm{mmHg}$ and/or diastolic blood pressure $\geq 95 \mathrm{~mm} \mathrm{Hg}$ in persistent hypertension or in gestational hypertension, chronic hypertension with superposed gestational hypertension or hypertension with subclinical organ damage with systolic arterial pressure $\geq 140 \mathrm{mmHg}$ and/ or diastolic pressure $\geq 90 \mathrm{~mm} \mathrm{Hg}$.

e) Decision of delivery should not be based on the degree of proteinuria f) Use of magnesium sulfate is recommended for severe preeclampsia, eclampsia or HELLP syndrome [8].

\section{Take Home Messages}

a) Hypertensive disorders during pregnancy are a leading cause of maternal and perinatal mortality worldwide.

b) Pre-eclampsia complicates $2 \%$ to $8 \%$ of pregnancies worldwide. In the USA, preeclampsia rates increased by $25 \%$ between 1987 and 2004. Preeclampsia treatment requires great resources.

c) Accepted are current clinical guidelines Bulletin № 212/2019 of ACOG for algorithm for diagnosing, treatment of gestational hypertension and preeclampsia, time of delivery and the use of magnesium sulfate.

d) Pregnantwomen with moderate to high risk for developing preeclampsia should receive $81 \mathrm{mg}$ of aspirin, and ideally it should be started between 12 and 16 weeks of gestation.

e) Treatment of gestational hypertension is at the same time and the treatment of preeclampsia, these two states can be regarded as a general spectrum of the same disease

f) ACOG also stresses that it is a priority to immediately treat the emergent hypertensive conditions, rather than to wait four hours to put the diagnose according to previous recommendations.

g) Non-steroid anti-inflammatory agents are now recommended as a first line treatment of pain postpartum over opioides, because they don't affect the values of the arterial pressure.

h) ACOG continues to recommend quantitatively determination of protein in urine for 24 hour or protein/ creatinine ratio; but if these tests are not available, proteinuria should be diagnosed as $2+$ per test strip instead of $1+$.

\section{Conclusion}

Hypertensive disorders in pregnancy are one of the most frequent reasons for maternal and perinatal mortality worldwide. Preeclampsia complicates $2-8 \%$ of pregnancies worldwide. Although current changes of guidelines for clinical judge on pregnancy and hypertension are not yet cardinal, they need to help treatment updates of preeclampsia and related pregnancy hypertensive conditions. The latest newsletter with guidelines for behaviour of ACOG in 2019 on "Pregnancy and Cardiovascular Disease" provides a clear recommendations focusing on the right time treatment of chronic hypertension, gestational hypertension, preeclampsia/eclampsia, pregnancy and acute hypertensive conditions, as well as treatment of pain postpartum in women with arterial hypertension.

\section{References}

1. (2019) Pregnancy and Heart Disease (Joint with the Presidential Task Force on Pregnancy and Heart Disease). Obstet Gynecol 133(5): e320356. 
2. (2019) Newsletter of practice of ACOG No.202: Gestational hypertension and preeclampsia. Obstet Gynecol 133(1): e1-25.

3. (2019) Newsletter of practice of ACOG No.203: Chronic hypertension during pregnancy. Obstet Gynecol. 133(1): e26-50.

4. Benjamin EJ, Virani SS, Callaway CW, Chamberlain AM, Chang AR, et al. (2018) Heart disease and stroke statistics-2018 update: a report from the American Heart Association. American Heart Association Council on Epidemiology and Prevention Statistics Committee and Stroke Statistics Subcommittee Circulation 137(12): e67-e492.

ISSN: 2574-1241

DOI: 10.26717/BJSTR.2019.23.003861

A Nikolov. Biomed J Sci \& Tech Res

(C) (P) This work is licensed under Creative

Submission Link: https://biomedres.us/submit-manuscript.php
5. Creanga AA, Syverson C, Seed K, Callaghan WM (2017) Pregnancyrelated mortality in the United States, 2011-2013. Obstet Gynecol 130(2): 366-373.

6. Elkayam U, Goland S, Pieper PG, Silverside CK (2016) High-risk cardiac disease in pregnancy: part I. J Am Coll Cardiol 68(4): 396-410.

7. Melchiorre K, Sharma R, Thilaganathan B (2014) Cardiovascular implications in preeclampsia: an overview. Circulation 130(8): 703-714.

8. (2018) ACOG Committee Opinion No.736: Optimizing postpartum care. Obstet Gynecol 131(5): e140-150.

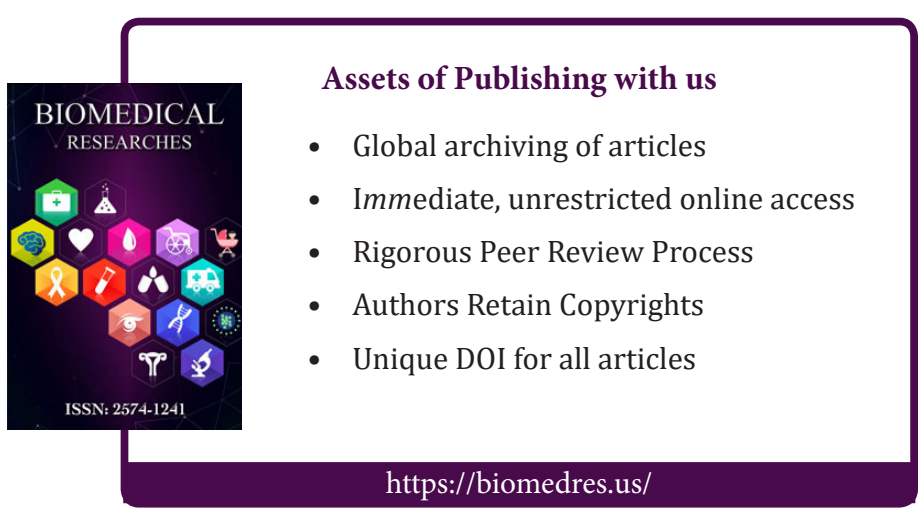

